\title{
Dysregulation of the Wnt Signaling Pathway and Synovial Stem Cell Dysfunction in Osteoarthritis Development
}

\author{
Junjie Huang, Chuanshun Chen, Chi Liang, Pan Luo, Guang Xia, Lina Zhang, \\ Xinxing Wang, Zi Wen, Xu Cao, and Song Wu
}

Stem cell dysfunction and failure have been found in joints afflicted by osteoarthritis (OA). However, the exact factors in the OA microenvironment that impair stem cell functions and the role of stem cell dysfunction in OA development have not been fully clarified. In this study, we evaluated the functional status of synovial mesenchymal stem cells (SMSCs) from OA patients and explored the influence of OA-SMSCs on cartilage degradation in a rat model. We then screened $138 \mathrm{Wnt}$ signaling-related genes in the synovium of OA patients, focusing on the effects of five WNT ligands on SMSC functions. The OA synovium showed mild hyperplasia, and we found a large number of $\mathrm{CD} 90^{+} / \mathrm{CD} 105^{+}$stem cells in synovial hyperplasia. The OA-SMSCs revealed a cellular senescence phenotype, with decreased proliferation and chondrogenic capacity, accompanied by enhanced migration, proinflammatory and matrix degradation activities. The intra-articular transplantation of these OA-SMSCs significantly aggravated the degradation and destruction of the articular cartilage. Of $138 \mathrm{Wnt}$ signaling genes, the expression of 86 genes was consistently altered in the OA synovium, among which the increased expression of $D V L 2, W N T 10 A$, and $D K K 3$ was the most marked. In general, we found that canonical $\mathrm{Wnt} / \beta$-catenin pathways were inhibited in the OA synovium, whereas noncanonical PCP and Wnt/Ca2 ${ }^{+}$pathways were activated. In vitro, WNT10A had an obvious antisenescence effect on SMSCs. WNT5B significantly inhibited the chondrogenic differentiation of SMSCs, and WNT10A and WNT5A increased the expression of inflammatory cytokines in SMSCs. In a rat model, WNT5A significantly aggravated joint degeneration, whereas WNT10A had a mild protective effect on cartilage integrity. In conclusion, stem cells in the OA synovium were functionally abnormal and promoted the development of OA, whereas dysregulation of the Wnt signaling pathway revealed a comprehensive influence on SMSC functions and cartilage degradation.

Keywords: synovial mesenchymal stem cells (SMSCs), Wnt signaling pathway, osteoarthritis, differentiation, cell senescence

\section{Introduction}

A RTICULAR CARTILAGE WAS previously believed to be incapable of self-repair. Over the past few years, however, spontaneous chondrogenesis has been observed in conditions such as joint offloading [1,2]. This ability to undergo cartilage repair is believed to be derived from residual stem cells in the joint, especially those residing in the synovial "stem cell pool" [3]. This finding has brought extensive attention to native stem cells in joints. These stem cells were found to be a critical cause of resistance against joint degeneration via their cell proliferation, migration, and differentiation [3]. However, the properties of stem cells are greatly influenced by their surrounding microenvironment [4].

The deteriorative intra-articular microenvironment resulting from senescent cell accumulation and chronic inflammation was found to alter the functions of intra-articular stem cells [5]. These stem cells, which are affected by osteoarthritis (OA), may not only exhibit an impaired capacity to repair damage [6] but also become a persistent degenerative factor in joints that accelerates the progression of OA. Therefore, it is extremely important to evaluate dysfunction in synovial mesenchymal stem cells (SMSCs) residing in OA joints (OA-SMSCs), clarify their role in joint degeneration, and, most importantly, explore the possible regulatory factors of SMSCs in the context of OA.

In recent years, Wnt signaling dysregulation has been increasingly found to play a role in age-related diseases, particularly OA [7-9]. Studies have indicated that disordered Wnt signaling in the synovium accelerates cartilage degeneration in OA [10,11]. Although the mechanism of this process is unclear, it has been found to be closely related to joint residual stem cell dysfunction [12]. Wnt signaling, a highly conserved pathway that determines multiple

Department of Orthopaedics, The 3rd Xiangya Hospital, Central South University, Changsha, China. 
embryonic development processes, has emerged as a critical regulator of adult stem cells in various tissues [13]. Wnt signaling disorder plays a critical role in stem cell failures, for example, hematopoietic stem cell senescence [14] and intestinal stem cell failure [15]. Based on this, regulators of Wnt signaling have been suggested as promising diseasemodifying osteoarthritis drugs (DMOADs) [12,16,17].

However, despite these findings, our understanding of the precise role of Wnt signaling in stem cells in OA joints is limited. Many issues remain to be elucidated. Wnt signaling is a complex process that involves at least 19 different ligands and 3 downstream pathways [18]. The specific effects of different Wnt ligands on OA-SMSCs and cartilage health remain unclear. Some Wnt ligands are likely beneficial to chondrogenesis and SMSC functions [19]. Disordered Wnt signaling may lead to or aggravate SMSC dysfunction and promote the progression of OA. In this study, we attempted to evaluate functional abnormalities of OA-SMSCs and their critical role in joint degradation. Further, we have presented a comprehensive screen for Wnt signaling-related genes in the OA synovium and explored the effects of five Wnt ligands on SMSC functions.

\section{Materials and Methods}

The study protocol was approved by the institutional review board of our institution (No. 2015-S056), and signed written consent was obtained from all study subjects. All experiments involving human tissues and animals were performed in accordance with guidelines approved by the IRB of Third Xiangya Hospital, Central South University (No. 2015-S056). Each sample was processed on signed informed consent.

\section{Synovium sample collection and SMSC identification}

Human synovium without obvious joint degeneration (Pre-OA synovium) was obtained during arthroscopic operation ( $n=5$, female, $58 \pm 7.6$ years of age). OA synovium was obtained during total knee arthroplasty $(n=5$, female, $59 \pm 4.9$ years of age, OA severity assessment shown in Supplementary Table S1). To extract RNA and protein, synovial samples were placed in liquid nitrogen within $10 \mathrm{~min}$. To determine SMSC localization, synovial samples were fixed with paraformaldehyde, dehydrated, embedded in paraffin, and cut into paraffinized sections for hematoxylin and eosin (HE) staining and immunofluorescence to detect CD90, CD105 (BioGems), and DAPI. To isolate SMSCs, synovial samples were washed three times with phosphate buffered saline (PBS), ground into $1-2 \mathrm{~mm}^{3}$ pieces, placed on $60-\mathrm{mm}$ culture dishes, and digested with $0.3 \%$ collagenase type I (Sigma) in Dulbecco's modified Eagle's medium (DMEM) (10567014; Gibco) with penicillin (100 U/mL), streptomycin $(100 \mu \mathrm{g} / \mathrm{mL}, 15140122 ; \mathrm{Gibco})$, and $10 \%$ fetal bovine serum (FBS) (10270106; Gibco) for $3 \mathrm{~h}$ in a humidified atmosphere of $5 \% \mathrm{CO}_{2}$ and $95 \% \mathrm{O}_{2}$ at $37^{\circ} \mathrm{C}$. The cells were passed through a 70- $\mu \mathrm{m}$ nylon cell strainer and collected by centrifugation at $250 \mathrm{~g}$ for $5 \mathrm{~min}$. The cells were then resuspended and plated in a $60-\mathrm{mm}$ diameter culture dish, with the medium replaced every 3 days.
Flow cytometry and multipotential differentiation assays were used to identify SMSCs. Antibodies against CD14, CD73, CD90, and CD105 (BioGems) were used for flow cytometry. A total of $1 \times 10^{5}$ passage 3 cells were suspended in $500 \mu \mathrm{L}$ PBS containing $20 \mu \mathrm{g} / \mathrm{mL}$ antibody. The cells were washed three times with PBS and suspended in $1 \mathrm{~mL}$ PBS for analysis after their incubation for $30 \mathrm{~min}$ at $4{ }^{\circ} \mathrm{C}$. Analyses were then performed via flow cytometry (BD FACSCalibur). Passage 3 SMSCs were plated in six-well plates $\left(2 \times 10^{5} /\right.$ well $)$, cultured for 14 days in OriCell osteogenic or adipogenic culture medium (Cyagen), and stained with alizarin red $\mathrm{S}$ or an oil red solution for osteogenesis or adipogenesis studies, respectively. A total of $2 \times 10^{5}$ passage 3 SMSCs were transferred into a $15-\mathrm{mL}$ conical tube (after their centrifugation into pellets), cultured for 21 days in OriCell chondrogenic culture medium (Cyagen), and stained with Alcian blue for chondrogenesis studies.

\section{SMSC dysfunction assessment}

Passage 3 SMSCs isolated from five Pre-OA or five OA patient synovial samples were cultured for cell function assessment.

Senescence-associated $\beta$-galactosidase staining. SMSCs were stained with a Senescence Detection Kit (K320-250; BioVision) according to the manufacturer's protocol after being fixed with $4 \%$ paraformaldehyde. The cells in six random fields per well were then counted.

EdU incorporation assay. Proliferating cells were determined with a Click-iT Plus EdU Alexa Fluor 488 Imaging Kit (Invitrogen) according to the manufacturer's protocol, and cell nuclei were stained with Hoechst 33342 (Invitrogen). The cells in six random fields per well were counted. The percentage of EdU-positive cells was calculated by using Image Pro-Plus version 6.0 for Windows (Media Cybernetics).

SMSC colony-forming unit $(C F U-F)$ assay. SMSCs were collected and counted, and the 1,000 cells were plated on a 60-mm culture dish with DMEM supplemented with penicillin $(100 \mathrm{U} / \mathrm{mL})$, streptomycin $(100 \mu \mathrm{g} / \mathrm{mL})$, and 10\% FBS. The medium was changed every 3 days. After 14 days, the cells were fixed with $4 \%$ paraformaldehyde and stained with $0.1 \%$ crystal violet.

Chondrogenic differentiation assay. SMSCs were cultured as cell pellets $\left(2 \times 10^{5}\right.$ /pellets $)$ in incomplete chondrocyte differentiation medium (iCDM) consisting of high-glucose DMEM (containing L-glutamine and sodium pyruvate), $1 \%$ ITS + Premix, $40 \mu \mathrm{g} / \mathrm{mL}$ proline, $100 \mathrm{nM}$ dexamethasone, and $50 \mu \mathrm{g} / \mathrm{mL}$ ascorbic acid-2-phosphate; they were treated with $20 \mathrm{ng} / \mathrm{mL}$ TGF- $\beta 3$ (PeproTech) in 15-mL conical tubes for 14 days. The diameters of the cell pellets were measured. For immunohistochemical assessment, the pellets were fixed, embedded in paraffin, cut into 4- $\mu \mathrm{m}$ sections, and incubated with rabbit polyclonal anti-COL I (14695-1AP; Proteintech, China), anti-COL II (Abcam), and antiCOL X (bs-0554R; Bioss, China) antibodies overnight at $4^{\circ} \mathrm{C}$. Secondary antibodies were detected by using an SABC-POD kit (Boster, China) according to the manufacturer's protocol. The protein expression of COL I, COL II, and COL $\mathrm{X}$ was then detected by western blotting (see the Western blotting section of the Materials and Methods section). The expression of COL I, COL II, COL X, IL-1 $\beta, I L-6$, 
$M M P-1$, and $M M P-13$ was detected by real-time quantitative polymerase chain reaction (RT-qPCR) (see the Realtime-qPCR section of the Materials and Methods section).

Cell migration assay. Cell migration assays were performed by using six-well Transwell plates with polycarbonate membranes with an $8-\mu \mathrm{m}$ pore size (Corning). Cells in DMEM were added to the upper chamber at a density of $1.0 \times 10^{6}(500 \mu \mathrm{L} /$ well $)$, and the lower chamber was filled with $10 \%$ FBS in culture medium. Cells that did not penetrate the membrane were removed with a cotton swab. Then, cells were stained with $0.1 \%$ crystal violet and counted in six random fields per well.

\section{Intra-articular transplantation of SMSCs in a rat OA model}

Pre-OA-SMSCs and OA-SMSCs were derived from six rats (6 months old, SD, female) each (12 rats in total) 1 and 8 weeks after development of the Hulth's model [20], respectively. Cells in each group were pooled together, and then SMSCs $\left(5 \times 10^{5}\right.$ in $100 \mu \mathrm{L}$ PBS, infected with lentivirusexpressing luciferase) were intra-articularly (i.a.) injected into the right knees of rats (14 rats in total, 6 months old) 1 week after development of the Hulth's model (illustrated in Fig. 3a). Bioluminescence imaging (BLIS; Perkin Elmer) was used to observe injected SMSC survival over 7 days. Three weeks after SMSC injection, the rats were sacrificed, and the right knee joints were collected. The total right knee joints of rat were fixed and flexed $\sim 60^{\circ}$; the knee was embedded in paraffin facing downward, cut coronally into 4- $\mu$ m-thick sections, and stained with Safranin O/fast green for OA assessment or used to detect the expression of Il-1 $\beta$, Il-6, Mmp-1, and Mmp-13 by RT-qPCR (see the Real-timeqPCR section of the Materials and Methods section). Four rats in each group were used for the experiment, and three sections of each knee were used for staining and analysis. The Osteoarthritis Research Society International (OARSI) score was performed by five orthopedic specialists.

\section{Wnt signaling pathway screening}

A qPCR array comprising 138 Wnt signaling-related genes was constructed in human OA and Pre-OA synovium by Tsingke (Beijing, China). Gene ontology (GO) and Kyoto Encyclopedia of Genes and Genomes (KEGG) pathway enrichment analyses of target genes were performed by NetworkAnalyst (www.networkanalyst.ca) according to the manufacturer's protocol [21]. The expression of $19 \mathrm{Wnt}$ ligands and $\beta$-catenin was further determined by RT-qPCR (see the Real-time-qPCR section of the Materials and Methods section). The expression of WNT5A, WNT5B, WNT7B, WNT9A, WNT10A, $\beta$-catenin, glycogen synthase kinase-3 $\beta$ (GSK-3 $\beta$ ) and p-GSK-3 $\beta$, c-Jun N-terminal kinase (JNK) and $\mathrm{p}-\mathrm{JNK}$, calmodulin-dependent kinase II (CaMKII) and p-CaMKII, protein kinase $\mathrm{C}$ (PKC), and p-PKC at the protein level was detected by western blotting (see the Western blotting section of the Materials and Methods section). Primary Pre-OA SMSCs and OA SMSCs were fixed in $4 \%$ paraformaldehyde and blocked in $5 \%$ bovine serum albumin (BSA) diluted in $0.5 \%$ Triton X-100. Then, the cells were incubated with rabbit polyclonal anti- $\beta$-catenin (CST) antibodies overnight at $4^{\circ} \mathrm{C}$. The cells were then incubated with a fluorophore-conjugated secondary antibody for $1 \mathrm{~h}$. Nuclei were counterstained with DAPI.

\section{Effects of WNT ligands on SMSCs}

We selected WNT5A, WNT5B, WNT7B, WNT9A, and WNT10A, the expression of which was shown to be increased by our results, for SMSC function assessment. Passage 3 SMSCs were cultured in iCDM containing $20 \mathrm{ng} / \mathrm{mL}$ WNT5A, WNT5B, WNT7B, WNT10A (Proteintech), WNT9A (Abnova), and TGF- $\beta 3$ (PeproTech). After 14 days of culture, the SMSCs were stained with a Senescence Detection Kit (K320250; BioVision) or assessed for $p 16^{I N K 4 a}$ and $p 21^{\text {Cip } 1}$ expression by RT-qPCR (see the Real-time-qPCR section of the Materials and Methods section). Cells were fixed in $4 \%$ paraformaldehyde and blocked in 5\% BSA diluted in $0.5 \%$ Triton X-100. Then, the cells were incubated with rabbit polyclonal anti-COL I (14695-1-AP; Proteintech), anti-COL II (Abcam), anti-COL X (bs-0554R; Bioss), and anti-Aggrecan (13880-1-AP; Proteintech) antibodies overnight at $4^{\circ} \mathrm{C}$. The cells were then incubated with a fluorophore-conjugated secondary antibody for $1 \mathrm{~h}$. Nuclei were counterstained with DAPI. The cells were also stained with Safranin O according to the manufacturer's protocol. The expression levels of SOX-9, COL II, Aggrecan (for chondrogenic differentiation), COL X, PTH1R, IHH, $M E F 2 C$ (for hypertrophy), SPP1, ALPL, RUNX2 (for osteogenesis differentiation), and $I L-1 \beta, I L-6, M M P-1, M M P-13$ (for cartilage degradation) were detected by RT-qPCR (see the Real-time-qPCR section of the Materials and Methods section). The expression levels of COL I, COL II, COL X, and Aggrecan were further detected by western blotting (see the Western blotting section of the Materials and Methods section).

\section{Real-time-qPCR}

RT-qPCR was conducted by using ChamQ Universal SYBR qPCR Master Mix (Vazyme, China) according to the manufacturer's protocol. An initial denaturation step was carried out at $95^{\circ} \mathrm{C}$ for $15 \mathrm{~min}$, followed by 40 cycles of denaturation at $95^{\circ} \mathrm{C}$ for $10 \mathrm{~s}$, annealing at $56^{\circ} \mathrm{C}$ for $30 \mathrm{~s}$, and extension at $72^{\circ} \mathrm{C}$ for $30 \mathrm{~s}$. The primer sequences are shown in Table 1. Gene transcriptional levels were normalized to those of GAPDH/Gapdh and calculated by using the $\lg 2^{-\triangle \triangle \mathrm{Ct}}$ method (Table 1).

\section{Western blotting}

Protein extracts were subjected to SDS-PAGE, and the proteins were transferred to PVDF membranes and blocked in blocking buffer (5\% skimmed milk) for $1 \mathrm{~h}$. The membranes were incubated overnight at $4^{\circ} \mathrm{C}$ with primary antibodies against WNT5A, WNT5B, WNT7B, WNT9A, WNT10A (Abcam), $\beta$-catenin (CST), GSK-3 $\beta$ and p-GSK3 $\beta$ (Servicebio, China), JNK and p-JNK (Abcam), CaMKII and p-CaMKII (Abcam), PKC and p-PKC (Abcam), COL I (14695-1-AP; Proteintech), COL II (Abcam), COL X (bs0554R; Bioss), aggrecan (13880-1-AP; Proteintech), and GAPDH. The next day, the membranes were incubated with fluorophore-conjugated secondary antibody at room temperature for $1 \mathrm{~h}$. Protein bands were imaged with an enhanced LI-COR Odyssey infrared imaging system (LI-COR Corp., NE). The protein levels were normalized to GAPDH levels. 
Table 1. Primer Sequences for Real-Time Quantitative Polymerase Chain Reaction

\begin{tabular}{|c|c|c|}
\hline Gene & Forward primer & Reverse primer \\
\hline WNT1 & TGATACGCCAAAATCCGGGG & GACGATCTTGCCGAAGAGGT \\
\hline$W N T 2$ & GGATGACCAAGTGTGGGTGT & GGTCATGTAGCGGTTGTCCA \\
\hline$W N T 2 B$ & GGGGCACGAGTGATCTGTG & GCATGATGTCTGGGTAACGCT \\
\hline WNT3 & GGAGAGGGACCTGGTCTACTA & CTTGTGCCAAAGGAACCCGT \\
\hline$W N T 3 A$ & TCTACGACGTGCACACCTG & CCTGCCTTCAGGTAGGAGTT \\
\hline WNT4 & CCATGAGTCCCCGCTCGT & CCAGGTACAGCCAGTTGCTC \\
\hline WNT5A & ATTCTTGGTGGTCGCTAGGTA & CGCCTTCTCCGATGTACTGC \\
\hline WNT5B & CTTTGGCTCGGAAACGGTG & GGCTCCAGTGGAATCTCTCT \\
\hline WNT6 & CGGGGAGCGTTTAAAGGACA & TTTATTGATACTAACCTCACCCACC \\
\hline$W N T 7 A$ & CGTCTCGCACACTTGCACC & CCGCGCTTTCCGGTTCATA \\
\hline$W N T 7 B$ & ACAAGTGCGGACACATTGG & AAGCCCGGTTGAGCGAC \\
\hline WNT8A & GAACCTGTTTATGCTCTGGGC & CAGCGTTCCCAAGCAAACTG \\
\hline$W N T 8 B$ & AAGTACCACGCAGCACTCAA & GTTCTCCAGGCAGTAGTCCG \\
\hline WNT9A & GAGCGCGATGGTCGGC & GTCGCAGGCCTTGTAGTGC \\
\hline WNT9B & GGAGATGCTAGAGGGCGCA & GGACAGCTTCAGCAGGTCAC \\
\hline WNT10A & TGCTCCTGTTCTTCCTACTGC & GGGGATCTTGTTGCGAGTCT \\
\hline WNT1OB & ATCCTCAAGCGCGGTTTCC & AAACTCTTGCCTCGGGACAG \\
\hline WNT11 & GACCTCAAGACCCGATACCTG & TAGACGAGTTCCGAGTCCTTC \\
\hline WNT16 & CTACAGCTCCCTGCAAACGA & CCAAGTTATCCCTCGCCCTC \\
\hline CTNNB1 & GTCTGAGGAGCAGCTTCAGT & TGGTAGTGGCACCAGAATGG \\
\hline$I L-1 \beta$ & CAGAAGTACCTGAGCTCGCC & AGATTCGTAGCTGGATGCCG \\
\hline$I L-6$ & CCTGAACCTTCCAAAGATGGC & TTCACCAGGCAAGTCTCCTCA \\
\hline$M M P-1$ & AGTACTGGGCTGTTCAGGGA & TGTCCTTGGGGTATCCGTGT \\
\hline$M M P-13$ & TGAGAGGCTCCGAGAAATGC & CGTCAAGTTTGCCAGTCACC \\
\hline COLI & GTGCGATGACGTGATCTGTGA & CGGTGGTTTCTTGGTCGGT \\
\hline COLII & TTCССССТССТGСТCСAA & TCTGTCCCTTTGGTCCTGGT \\
\hline $\operatorname{COLX}$ & GGGGCTAAGGGTGAAAGGG & GGTCCTCCAACTCCAGGATCA \\
\hline SOX9 & AGGAAGTCGGTGAAGAACGG & AAGTCGATAGGGGGCTGTCT \\
\hline$A C A N$ & TGGTGATGATCTGGCACGAG & CGTTTGTAGGTGGTGGCTGT \\
\hline PTH1R & CTGGGCATGATTTACACCGTG & CAGTGCAGCCGCCTAAAGTA \\
\hline $\mathrm{IHH}$ & AACTCGCTGGCTATCTCGGT & GCCCTCATAATGCAGGGACT \\
\hline$M E F 2 C$ & ACCGACATGGACAAAGTGCT & GAACAGCCTCCACGATGTCT \\
\hline SPP1 & CTGACCCATCTCAGAAGCAGAA & TTACTTGGAAGGGTCTGTGGG \\
\hline$A L P L$ & CAGAAGCTCAACACCAACGTG & TGTGGAGACACCCATCCCATC \\
\hline$R U N X 2$ & GGTGTTCCAAAGACTCCGGC & GGAATACGCATCACAACAGCC \\
\hline GAPDH & AATGGACAACTGGTCGTGGAC & CССTCCAGGGGATCTGTTTG \\
\hline$I l-1 \beta$ & GGCTTCCTTGTGCAAGTGTC & TGTCGAGATGCTGCTGTGAG \\
\hline$I l-6$ & CTCCGCAAGAGACTTCCAGC & TCTGACAGTGCATCATCGCT \\
\hline Mmp-1 & ATGAGACGTGGACCGACAAC & TGAGTGAGTCCAAGGGAGTG \\
\hline Mmp-13 & GCAGCTCCAAAGGCTACAAC & TCTGGTGTTTTGGGGTGCTT \\
\hline Gapdh & GGAGCGAGATCCCGTCAAGA & TCGTGGTTCACACCCATCAC \\
\hline
\end{tabular}

\section{Effects of WNT ligands on joint degeneration}

Hulth's model of OA was used for the surgical induction of OA. One week after surgery, $20 \mu \mathrm{g} / \mathrm{mL}$ WNT5A, WNT5B, WNT7B, WNT10A (Proteintech), or WNT9A (Abnova) proteins dissolved in PBS and PBS (control) was injected into the articular cavity once a week for 1 month $(0.1 \mathrm{~mL}$ each time). Then, the knees were obtained, fixed in $4 \%$ paraformaldehyde, dehydrated with an ethanol gradient, embedded in paraffin, and sectioned, after which the sections were stained for histological evaluation by Safranin O/ fast green staining. The OARSI guidelines were used to evaluate the degree of OA [22].

\section{Statistical analysis}

Values in the text and figures are expressed as the mean $\pm S D$, unless otherwise noted. Statistical significance was determined by Student's $t$-test or analysis of variance
(ANOVA) by using SPSS 17.0 (SPSS, Inc., Chicago, IL). Differences in which $P<0.05$ were considered statistically significant.

\section{Results \\ SMSC localization and identification}

OA and Pre-OA synovial samples were stained with the mesenchymal stem cell markers CD90 and CD105. CD90 ${ }^{+}$ $\mathrm{CD}_{105^{+}}$stem cells were present mostly in the synovial lining, but a small population of cells resided in the sublining tissue (Fig. 1a). Unlike the Pre-OA synovium, the OA synovium showed mild hyperplasia. We found a large number of $\mathrm{CD} 90^{+} / \mathrm{CD} 105^{+}$stem cells in synovial hyperplasia (Fig. 1a). The isolated SMSCs displayed a typical spindle-like shape after 14 days of culture. The cells were successfully induced to undergo chondrogenic, osteogenic, and adipogenic differentiation. Cells did not express CD14 but did express CD73, CD90, and CD105 (Fig. 1b, c). 

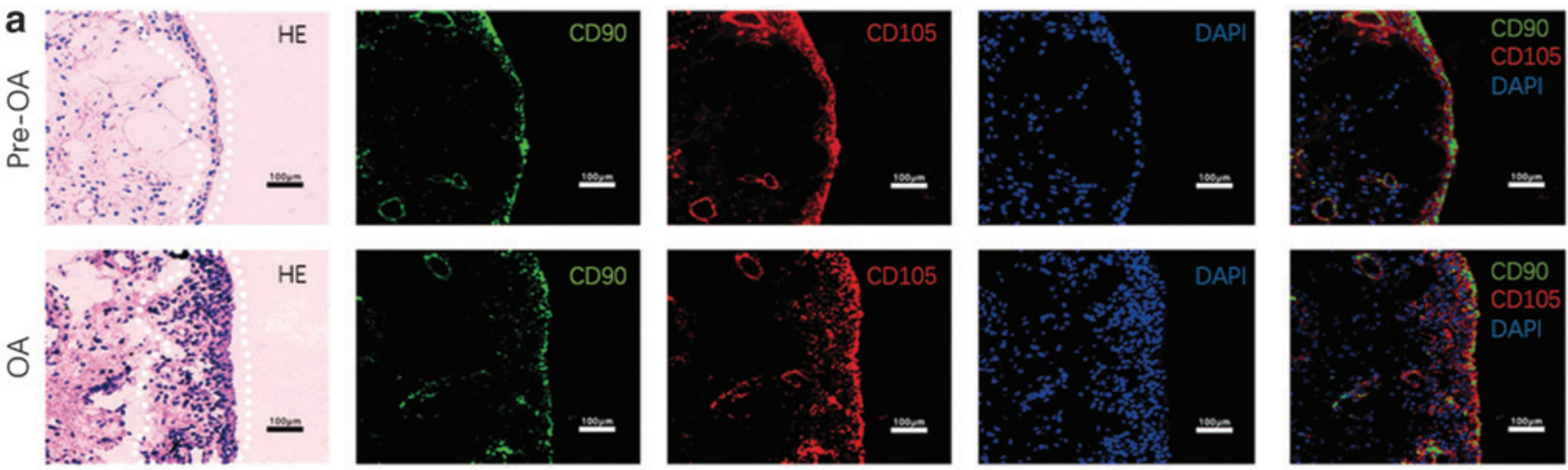

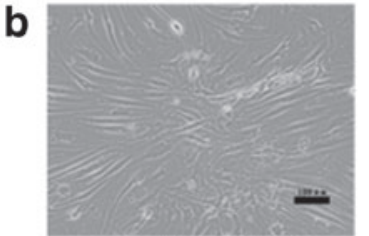

Normal

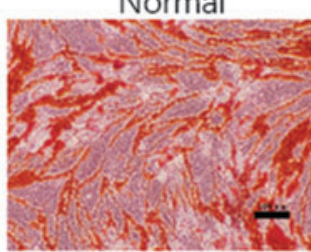

Osteogenesis

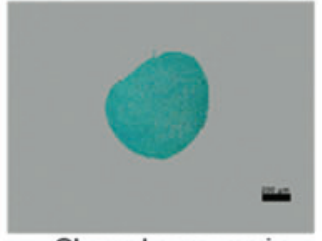

Chondrogenesis

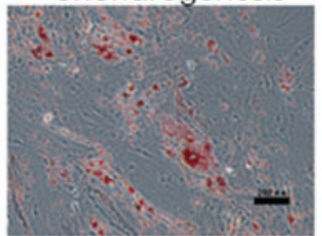

Adipogenesis
C
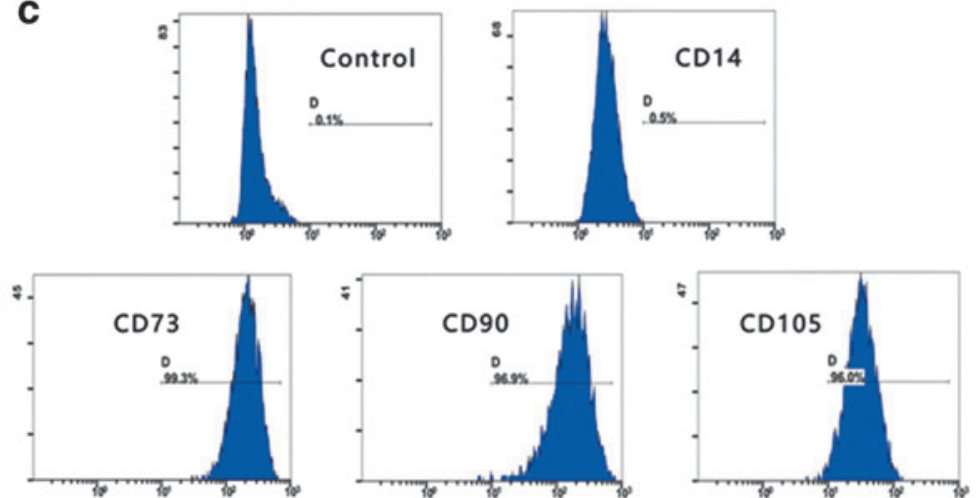

FIG. 1. The localization and identification of SMSCs in the human synovium. (a) HE staining and immunofluorescence staining of the Pre-OA (upper) and OA (lower) synovium for CD90 (green), CD105 (red), and DAPI (blue); scale bars $=100 \mu \mathrm{m}$. (b) The chondrogenesis, osteogenesis, and adipogenesis of SMSCs; scale bars $=100 \mu \mathrm{m}$. (c) Expression of the SMSC surface markers CD14, CD73, CD901, and CD105 determined by flow cytometry. OA, osteoarthritis; SMSCs, synovial mesenchymal stem cells.

\section{Functional evaluation of OA-SMSCS}

OA-SMSCs showed obvious cellular senescence. More than $30 \%$ of the OA-SMSCs were positively stained by SA- $\beta$-Gal, in contrast to only $3 \%$ of Pre-OA-SMSCs. Correspondingly, the proliferative activity of OA-SMSCs was lower than that of Pre-OA-SMSCs. Compared with Pre-OASMSCs, the percentage of Edu-positive OA-SMSCs was $80 \%$ lower, and the number of CFU-Fs was $77 \%$ lower (Fig. 2a). Meanwhile, the chondrogenic differentiation of OA-SMSCs was impaired. After induction with chondrogenic medium, OA-SMSC pellets were 39\% smaller in diameter than those formed by Pre-OA-SMSCs. OA-SMSC pellets contained more type I and type $X$ collagen (COL I/ $\mathrm{COL} \mathrm{X}$ ) and less type II collagen (COL II) than Pre-OASMSC pellets (Fig. 2b). In addition, production of the inflammatory cytokines $I L-1 \beta / 6$ and the matrix metalloproteinase MMP13 was increased in OA-SMSCs compared with Pre-OA-SMSCs. Likewise, the migration of OASMSCs was significantly increased (Fig. 2a, c).

\section{OA-SMSCs aggravate joint degeneration}

The i.a. injected SMSCs resettled and survived in the articular cavity for $>1$ week, and the survival rate of OASMSCs was slightly lower than that of Pre-OA-SMSCs (Fig. 3b). Pre-OA-SMSCs, to some extent, rescued surgery- induced joint degeneration. In contrast, OA-SMSCs significantly aggravated degradation and destruction of the articular cartilage (Fig. 3c). In addition, OA-SMSCs increased the expression of inflammatory cytokines and matrix metalloproteinases in the synovial membrane (Fig. 3c).

\section{Expression of Wnt signaling genes in the OA synovium}

Among 138 Wnt signaling genes, 116 genes were detected, and the expression of 86 genes was consistently altered (80 upregulated and 6 downregulated; $\log _{2} \mathrm{FC} \geq 1$ or $\leq-1$, respectively, Supplementary Table S2). Among these differentially expressed genes, the increased expression of DVL2, WNT10A, and DKK3 in the OA synovium was the most marked. Thirty-nine differentially expressed genes were associated with the canonical Wnt pathway, 25 genes were associated with the Wnt/planar cell polarity (PCP) pathway, and nine genes were associated with the $\mathrm{Wnt} / \mathrm{Ca}^{2+}$ modulation pathway (Fig. 4a). The potential functions of these 86 genes in Wnt signaling pathways were analyzed through GO annotation and KEGG analyses. The most enriched GO biological process terms were "regulation of signal transduction" and "tissue development." The most enriched GO cellular component terms were "extracellular space" and "proteinaceous extracellular matrix." The most 

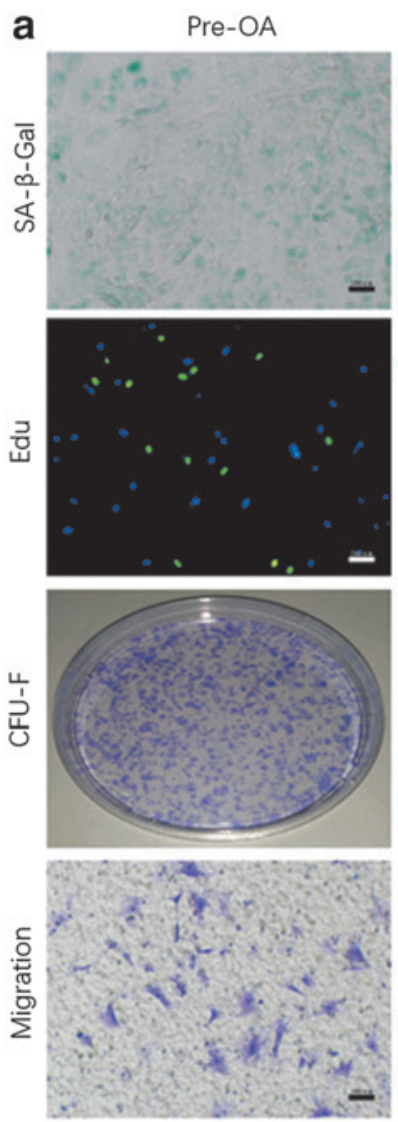

$\mathrm{OA}$
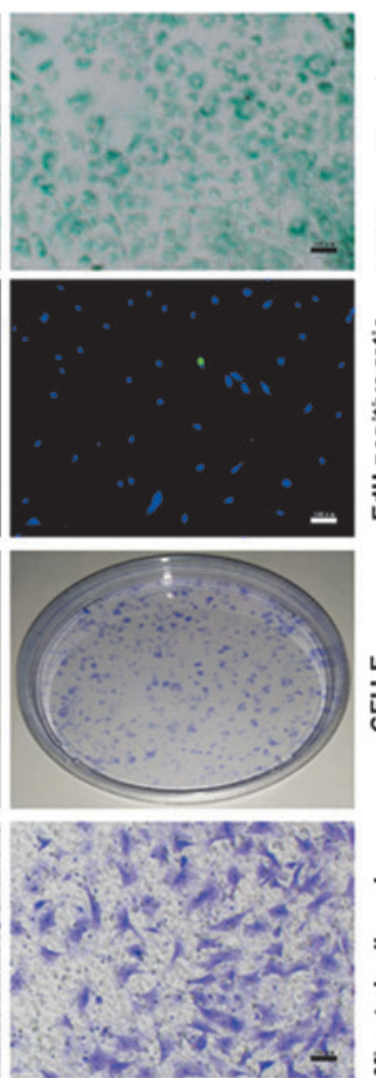
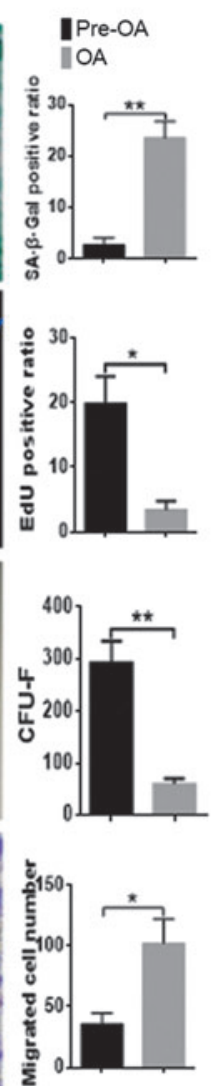
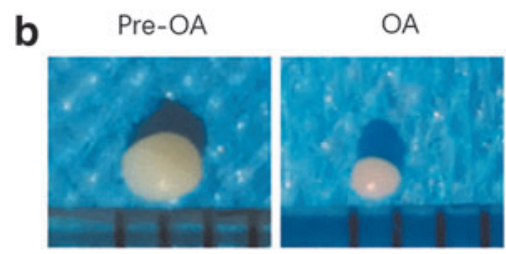

Pre-OA
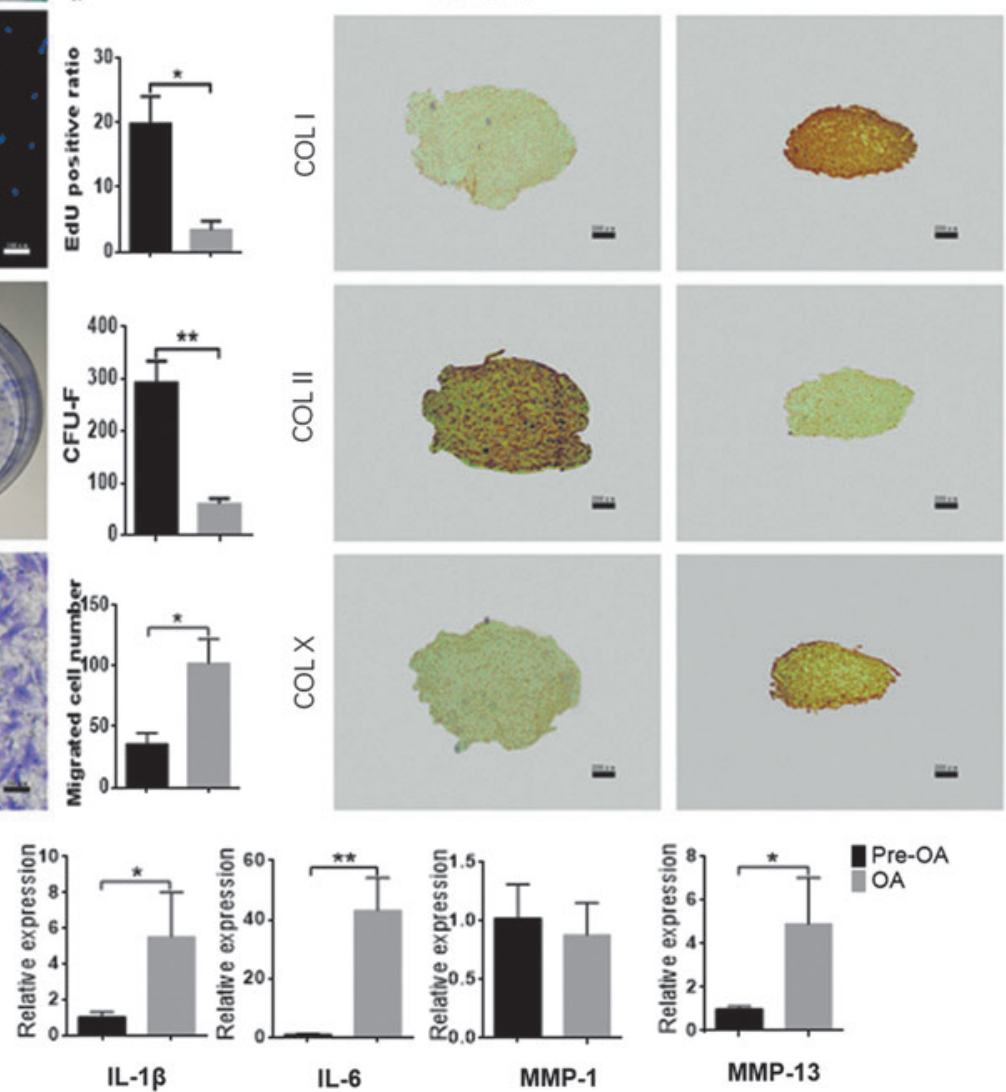

FIG. 2. Functional evaluation of SMSCs in vitro. (a) Primary SMSCs from Pre-OA (left) and OA (right) patients were stained for SA- $\beta-G a l, E d U$, and CFU-Fs. The ratios of SA- $\beta$-Gal-positive and EdU-positive (green) cells were calculated $(n=5)$; in addition, the number of colonies was calculated by the CFU-F assay $(n=5)$. Transwell assays were used to assess cell migration, which is reported in terms of the number of migrated cells $(n=5)$. (b) The gross appearance (upper) of PreOA-SMSCs (left) and OA-SMSCs (right) and immunohistochemical (lower) staining for COL I, COL II, and COL X in PreOA-SMSCs (left) and OA-SMSCs (right) after 14 days of chondrogenic induction $(n=5)$. (c) The expression levels of $C O L$ I, COL II, COL X, IL-1 $\beta, I L-6, M M P-1$, and MMP-13 in Pre-OA-SMSCs and OA-SMSCs after 14 days of chondrogenic induction $(n=4)$. $* P<0.05, * * P<0.01$. CFU-F, colony-forming unit.

enriched GO molecular function terms were "positive regulation of transcription, DNA dependent" and "PDZ domain binding." The significantly enriched pathways identified by the KEGG pathway analysis were "Wnt signaling pathway," "Breast cancer," "Hippo signaling pathway," "Pathways in cancer," "Basal cell carcinoma," "Proteoglycans in cancer," "Melanogenesis," "Signaling pathways regulating pluripotency of stem cells," "mTOR signaling pathway," and "AGE-RAGE signaling pathway in diabetic complications" (Fig. 4a).

The expression of 19 WNT ligands in the OA synovium was further detected by RT-qPCR. Among those ligands, $W N T 7 B, W N T 9 A$, and WNT10A were the most highly expressed in the OA synovium (Fig. 4c). However, only WNT10A was significantly increased at the protein level (Fig. 4c). $\beta$-catenin (canonical Wnt pathway) nuclear trans- location decreased in OA-SMSCs, and $\beta$-catenin expression at the protein and mRNA levels and the ratios of p-GSK3 $\beta$ / GSK3 $\beta$ were decreased in the OA synovium; whereas the ratios of $\mathrm{p}-\mathrm{JNK} / \mathrm{JNK}$ (Wnt/PCP pathway) and p-CaMK II/ CaMK II, p-PKC/PKC (Wnt/Ca2 ${ }^{+}$pathway) were increased (Fig. 4b and Supplementary Fig. S1).

\section{Effects of WNT ligands on SMSCs}

We selected five WNT ligands that were highly expressed in the OA joint: WNT5A, 5B, 7B, 9A, and 10A. We found that these WNT ligands have very discrepant effects on SMSC functions. WNT5A and WNT7B induced senescence in SMSCs, whereas WNT10A had an obvious antisenescence effect (Fig. 5a). WNT ligands inhibited the chondrogenic differentiation of SMSCs to different degrees, and 
a

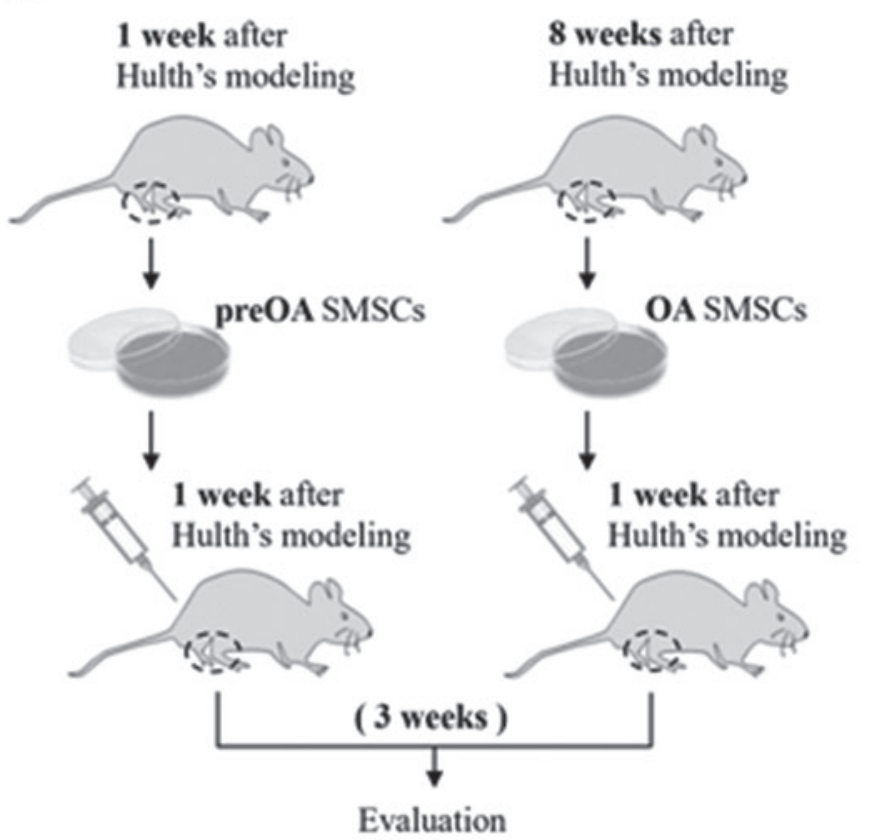

b

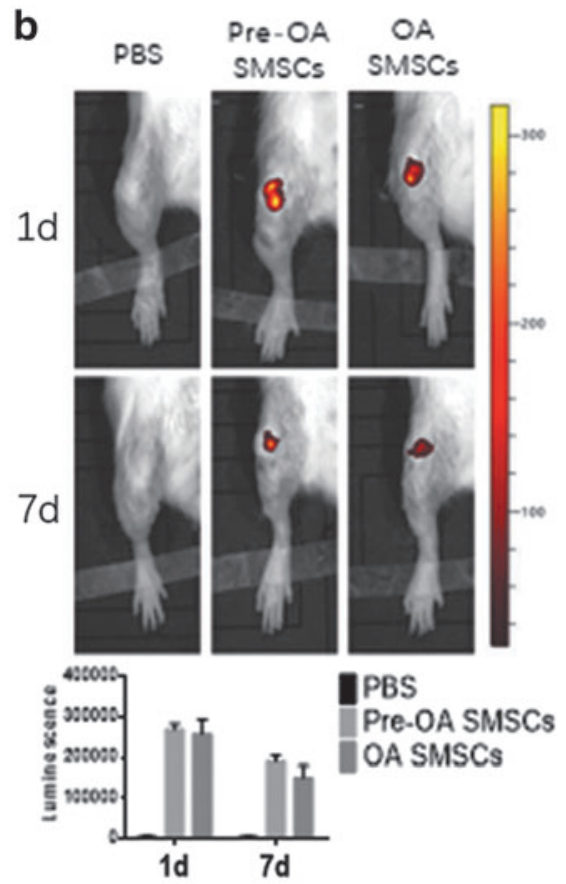

C
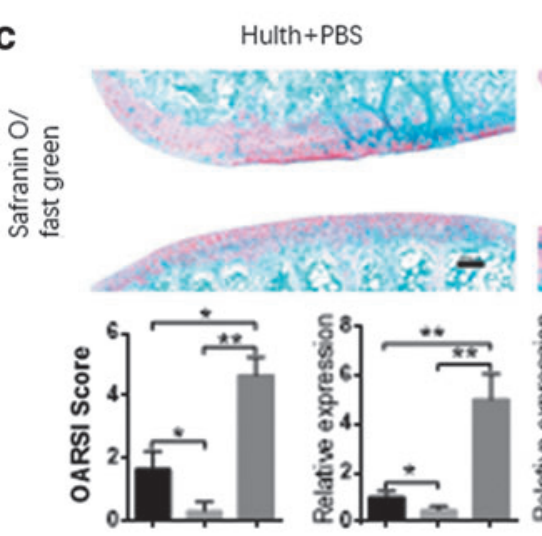

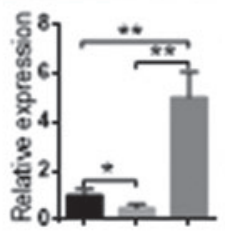

IL-1 $\beta$
Hulth+Pre-OA SMSCs
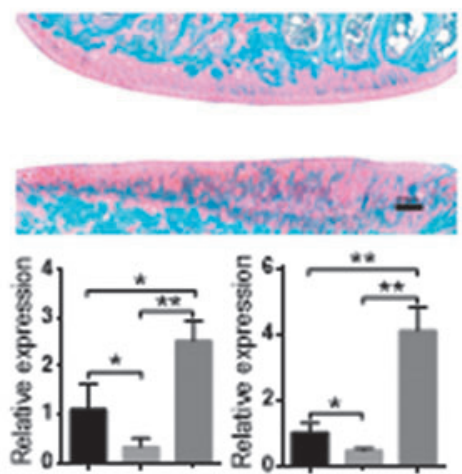

IL-6

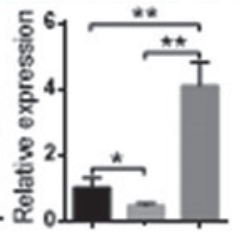

MMP-1
Hulth+OA SMSCs
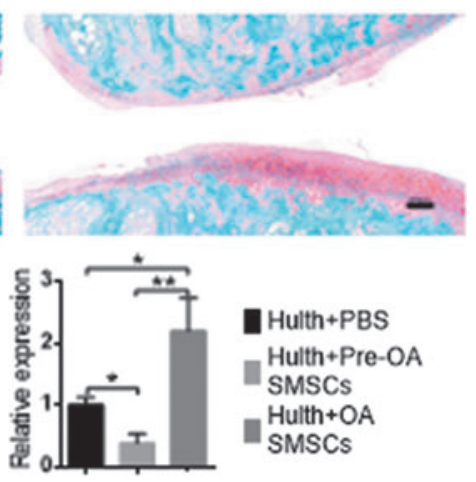

MMP-13

FIG. 3. OA-SMSC transplantation aggravates joint degeneration in rats after Hulth's modeling. (a) Schematic for the experiment in (b) and (c), Control group (PBS), $n=4$; Pre-OA-SMSCs group, $n=5$; OA-SMSCs group, $n=5$ (b) Representative luminescence images of rats (1 week after Hulth's modeling) on the first and seventh day after the intra-articular transplantation of SMSCs (upper) and quantification of luminescence (lower). (c) Safranin O/fast green staining was performed 3 weeks after transplantation (upper, left; scale bars $=200 \mu \mathrm{m}$ ), and joints were evaluated by the OARSI guidelines. Expression of $I l-1 \beta, I l-6, M m p-1$, and $M m p-13$ in the synovium 3 weeks after transplantation. $* P<0.05$, $* * P<0.01$. OARSI, Osteoarthritis Research Society International.

FIG. 4. Expression of WNT signaling genes in the OA synovium. (a) Significantly differentially expressed genes between the Pre-OA and OA synovium (left: canonical pathway, blue triangle; PCP pathway, red triangle; calcium modulation pathway, yellow triangle). KEGG pathway enrichment analysis and gene ontology analysis of significantly DEGs. (b) Immunofluorescence staining of the Pre-OA (upper, left) and OA (lower, left) SMSCs for $\beta$-catenin (red) and DAPI (blue); scale bars $=50 \mu \mathrm{m}$; expression of $\beta$-catenin detected by RT-qPCR; expression of $\beta$-catenin and ratio of p-GSK3 $\beta / G S K 3 \beta$; ratio of $\mathrm{p}-\mathrm{JNK} / \mathrm{JNK}$; ratio of $\mathrm{p}-\mathrm{CaMK}$ II/CaMK II and p-PKC/PKC detected by WB. (c) The protein expression of WNT5A, WNT5B, WNT7B, WNT9A, and WNT10A (upper) and mRNA expression of 19 WNT ligands (lower, left) in the Pre-OA and OA synovium. ${ }^{*} P<0.05$. CaMKII, calmodulin-dependent kinase II; DEG, differentially expressed genes; GSK-3 $\beta$, glycogen synthase kinase-3 $\beta$; JNK, c-Jun N-terminal kinase; KEGG, Kyoto Encyclopedia of Genes and Genomes; PCP, planar cell polarity; PKC, protein kinase C; RT-qPCR, real-time quantitative polymerase chain reaction, WB, western blotting. 


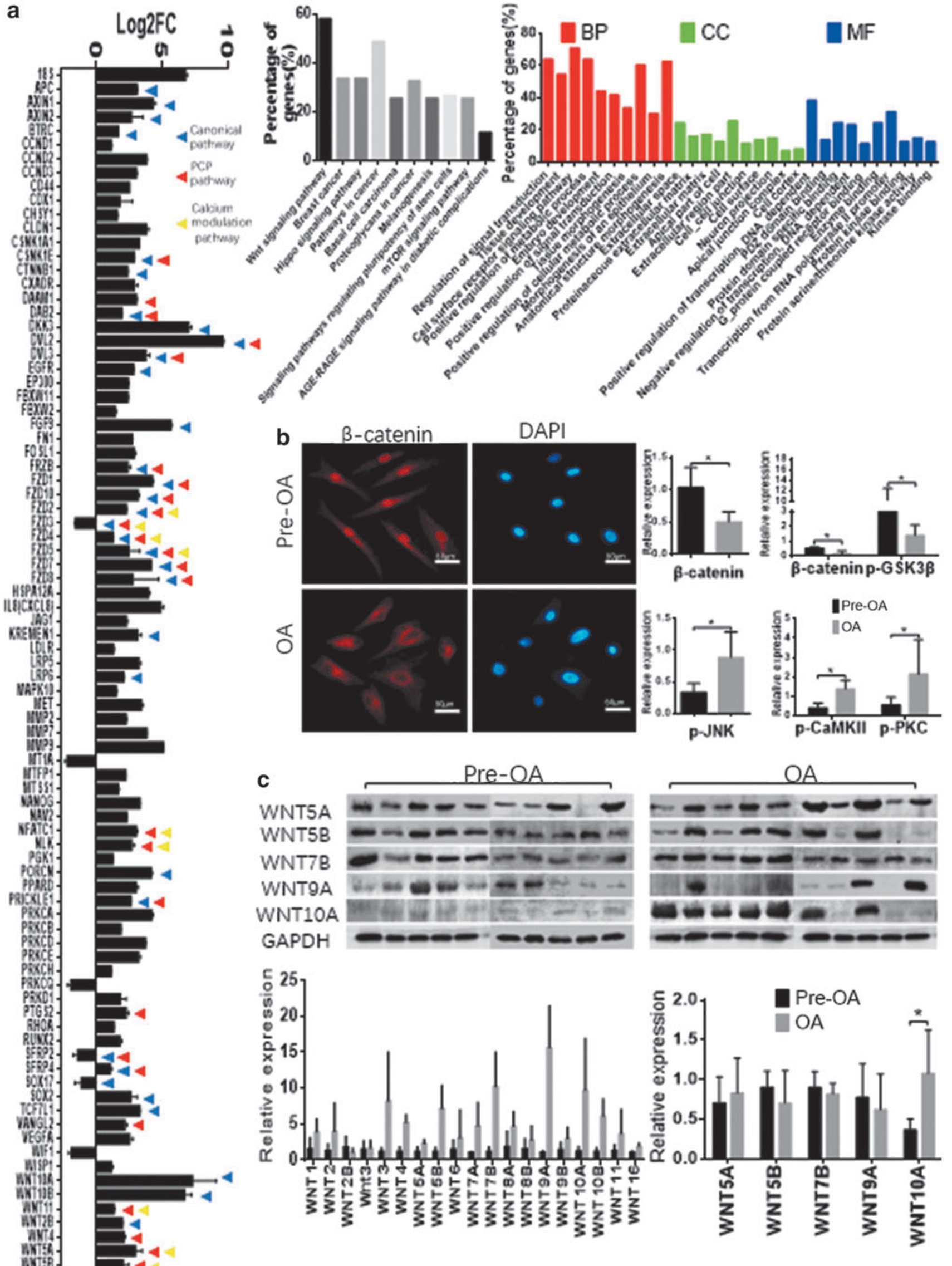


a
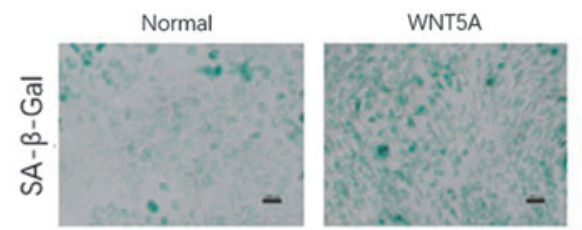

WNT5B

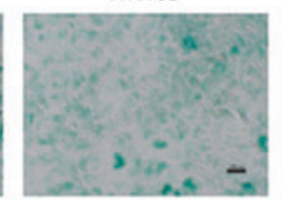

WNT7B

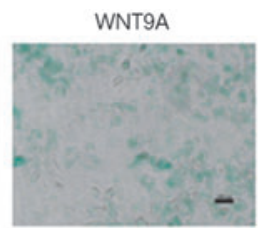

WNT10A
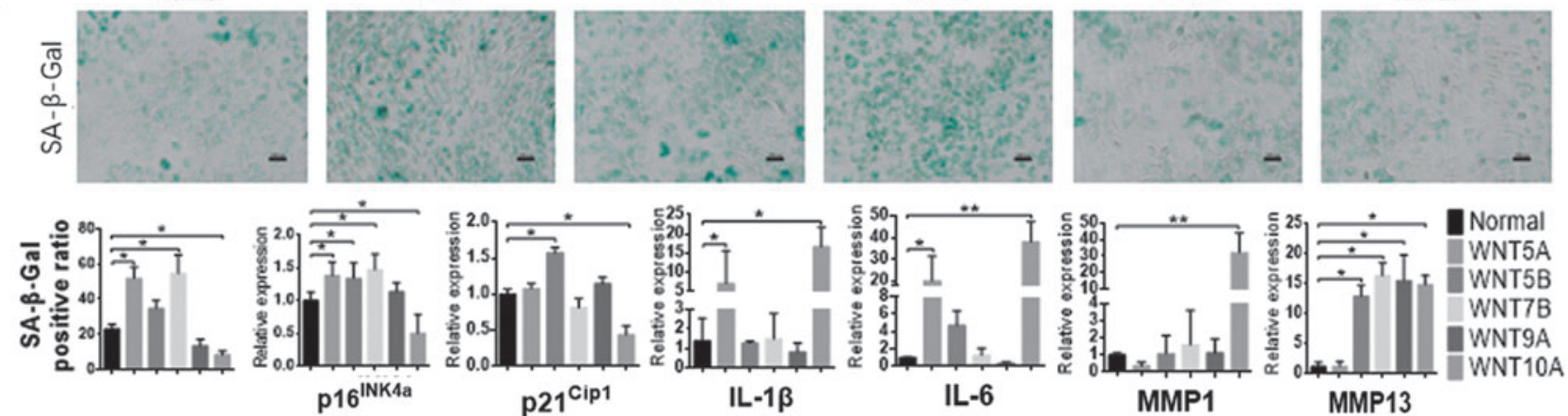

b
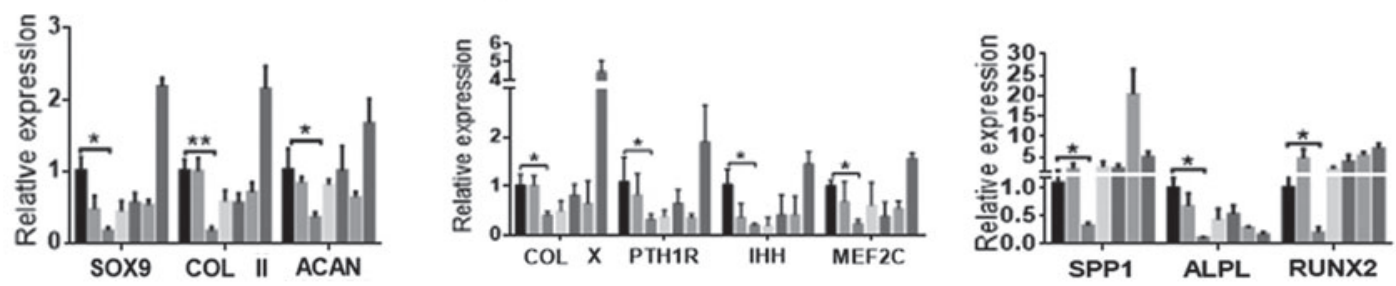

Normal WNT5A WNT5B WNT7B WNT9A WNT10A TGF- $\beta 3$
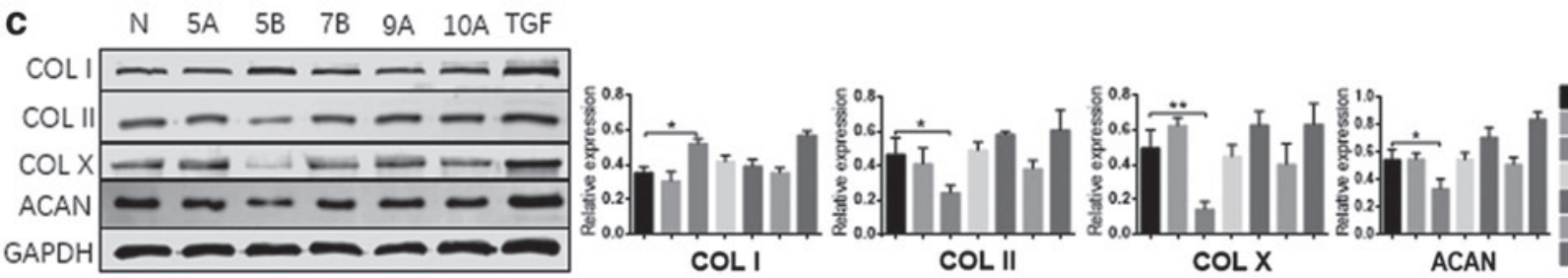

Normal WNT5A WNT5B WNT7B WNT9A

COLX

TGF. $\beta 3$

FIG. 5. Effects of WNT ligands on SMSCs in vitro. (a) Staining for SA- $\beta$-Gal and the ratio of SA- $\beta$-Gal-positive cells (upper, scale bars $=100 \mu \mathrm{m}, n=5)$ after 14 days of culture in iCDM supplemented with WNT5A, 5B, 7B, 9A, or $10 \mathrm{~A}(20 \mathrm{ng} / \mathrm{mL})$. The expression of $p 16^{\text {Ink } 4 a}, p 21^{\text {Cip } 1}, I L-1 \beta, I L-6, M M P-1$, and MMP-13 (lower). (b) The mRNA expression levels of SOX9, COL II, $A C A N$ (chondrogenic differentiation), COL X, PTH1R, IHH, MEF2C (hypertrophy), SPP1, ALPL, and RUNX2 (osteogenesis differentiation) (right). (c) The protein expression levels of COL I, COL II, COL X, and ACAN after culture for 14 days in conditional medium $(n=4)$. $* P<0.05, * * P<0.01$. iCDM, incomplete chondrocyte differentiation medium; ACAN, aggrecan.

WNT5B, which inhibited differentiation to the greatest degree, inhibited not only chondrogenic differentiation but also osteogenic differentiation and hypertrophy. In addition, WNT5B promoted fibrosis by increasing COL I expression in SMSCs (Fig. 5b, c and Supplementary Fig. S2). WNT10A and WNT5A significantly increased expression of the inflammatory cytokines $I L-1 \beta$ and $I L-6$ in SMSCs, whereas WNT10A also increased the expression of $M M P 1$. WNT5B, WNT7B, and WNT9A promoted MMP13 expression in SMSCs (Fig. 5a). The effects of the WNT ligands on SMSCs reflected their impact on joint degeneration to some extent. WNT5A significantly aggravated joint degeneration, whereas WNT5B and WNT7B also played a role in promoting joint degeneration. In contrast, WNT10A had a mild protective effect on cartilage integrity (Fig. 6).

\section{Discussion}

Roelofs et al. described a group of residual joint interzone cells in the synovium, surface layer of the meniscus, and cartilage expressing growth/differentiation factor 5 (Gdf5) [23]. These $\mathrm{Gdf5}^{+}$cells were found to possess the characteristics of mesenchymal stem cells (MSCs) and proliferate in response to joint injury to promote cartilage repair. We directly observed these MSCs in the synovial lining and sublining through the stem cell surface marker CD90/CD105 and found that they contribute to synovium hyperplasia in OA (Fig. 1a). However, SMSCs are actually a group of cells with a high degree of phenotypic heterogeneity. Currently, there are no widely recognized specific surface markers that can select the entire SMSC fraction. Instead, SMSCs are usually identified by the markers of MSCs derived from bone marrow or other tissues [3]. Gullo and De Bari identified a distinct SMSC subset with high chondro-osteogenic potency by using the surface markers CD73 and CD39 [24]. CD44, CD90, and CD105 have also been used to identify SMSCs [25]. We used CD73, CD90, and CD105 as surface markers in combination with multipotential differentiation to identify SMSCs in this study.

SMSCs are believed to be activated by joint degeneration and migrate through synovial fluid (SF). Jones et al. found that the number of MSCs in early OA SF was increased by 20-fold compared with rheumatoid arthritis (RA) SF [26] and 7-fold compared with normal SF [27]. The number of MSCs in the OA synovium were also shown to be increased [25]. We found that the ability of OA-SMSCs to migrate was significantly increased compared with control cells (Fig. 2a). SMSC activation is a key part of cartilage repair during acute cartilage injury. However, the persistent activation of SMSCs in a chronic degenerative environment is likely detrimental to joint health. Del Rey et al. described a subpopulation of CD271-expressing stem cells in the 
FIG. 6. Effects of WNT ligands on SMSCs in vivo. Safranin O/fast green staining was performed after the intra-articular transplantation of different Wnt ligands (once a week for 1 month) (left, bars $=200 \mu \mathrm{m}$ ), and joints were evaluated by the OARSI guidelines (right, $n=3)$. $* P<0.05$.
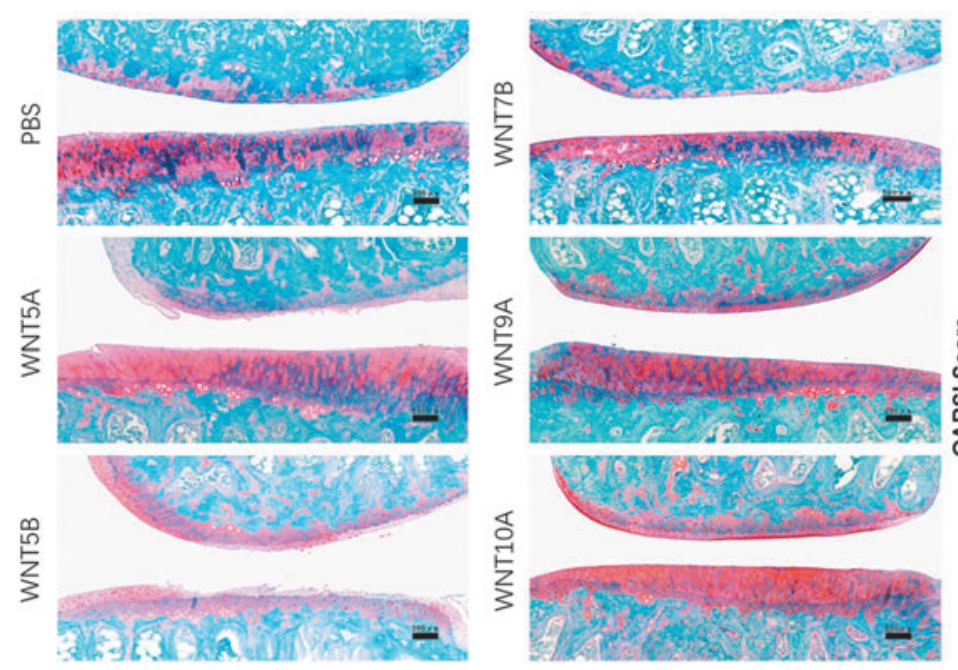

synovium. These CD271 ${ }^{+}$stem cells were markedly increased in OA and found to secrete large amounts of IL-6 and MMP-1/3 [28]. Several studies have explored the effect of OA SF on the secretion of inflammatory cytokines and MMPs in MSCs [29-31]. Leijs et al. demonstrated that OA SF had no effect on the expression of IL-6 and TNF $\alpha$ in MSCs [29]. However, OA SF was found to upregulate VEGF expression in MSCs and inhibit the expression of IL$1 \beta$ [30]. The effects of SF in OA at different stages are also inconsistent. Compared with late-stage OA SF, early-stage OA SF significantly increased IL-6 secretion in MSCs [31]. In this study, we directly compared the chondrodegradation of MSCs from the OA and Pre-OA synovium and found that the inflammatory cytokines IL-1 $\beta / 6$ and MMP13 were expressed at higher levels in OA-SMSCs than in Pre-OASMSCs (Fig. 2c). This finding is consistent with our observation of significant OA-SMSC senescence (Fig. 2a). Jeon et al. found that senescent cells accumulated in the OA synovium and demonstrated that these senescent cells play a key role in promoting the destruction of cartilage [32]. However, which cells in the synovium are senescent is unclear. Because of their persistent resistance to tissue degeneration, somatic stem cells tend to accumulate damage and become senescent [33]. Senescent SMSCs exhibit not only decreased proliferative activity but also a senescenceassociated secretory phenotype, as they continuously produce matrix-degrading enzymes and inflammatory cytokines. In addition, we found that the chondrogenic differentiation of OA-SMSCs was decreased, whereas the tendency to undergo fibrosis and hypertrophy increased (Fig. 2b, c). All of these factors may lead to the transformation of SMSCs from a factor that maintains joint health to one that accelerates joint degeneration (Fig. 3c).

WNT proteins are cysteine-rich secreted glycolipoproteins that regulate development, cell proliferation and motility, cell fate determination, and the generation of cell polarity $[34,35]$. Wnt signaling plays a vital role in regulating the proliferation and differentiation of MSCs [36], and the Wnt signaling pathway plays a unique role in joint tissues as it controls chondrocyte, osteoblast, and synovial cell functions [37]. In general, Wnt signaling can be divided into three pathways: a canonical $\mathrm{Wnt} / \beta$-catenin pathway and a noncanonical Wnt pathway, including the Wnt/PCP and
Wnt/Ca2 ${ }^{+}$pathway [38]. Meo et al. found that WNT5A and WNT7B were greatly increased in cartilage during spontaneous arthritis [39]. In addition, Tao et al. determined that the expression of WNT5A and WNT5B was increased in OA-SMSCs and OA-SMSC-derived exosomes [40]. Similarly, in some GEO datasets, we observed increased expression of WNT5A, 5B, and WNT7B in OA synovium (GSE1919, GSE46750, GSE55235, GSE55457, and GSE82107). WNT4, 5A, 5B, 6, 7A, 7B, and 11 were the Wnt ligands that preferentially activated the noncanonical Wnt pathway. Our study found that WNT4, 5A, 5B, 7B, and WNT11 were the main expressed noncanonical Wnt ligands in OA synovium (Fig. 4a, c). This finding is consistent with the results of other studies mentioned earlier, suggesting a dominant activation of the noncanonical Wnt pathway in the OA joint. We also found that WNT10A and $10 \mathrm{~B}$, as canonical Wnt ligands, showed significantly increased expression in partial OA synovium, which was not found in other studies. Therefore, we examined the key proteins in the three Wnt pathways in OA synovium, and, indeed, we found a significant decrease in the expression and nuclear translocation of $\beta$-catenin and the expression of phosphor-GSK $3 \beta$ (Wnt/ $\beta$-catenin pathway), as well as an increase in the expression of phosphor-JNK (Wnt/PCP pathway) and phosphor-CaMK II, phosphor-PKC (Wnt/ $\mathrm{Ca}^{+}$pathway). These results suggested the presence of a canonical to noncanonical Wnt signaling switch in OA synovium; the role of this switch in the pathogenesis of OA is still unknown. In generally, the $\mathrm{Wnt} / \beta$-catenin pathway is believed to be closely related to the development of OA, and inhibition of the $\mathrm{Wnt} / \beta$-catenin pathway is believed to slow OA progression. Lietman et al. determined that the expression of $\beta$-catenin was upregulated in the destabilization of the medial meniscus model of OA and human OA synovial fibroblasts, and inhibition of the $\mathrm{Wnt} / \beta$-catenin pathway with the $\beta$-catenin inhibitor XAV-939 was found to alleviate OA progression [41]. Hu et al. also found that OA cartilage exhibited higher levels of $\beta$-catenin mRNA and protein than normal cartilage [42]. However, Theologis et al. found that Dickkopf-related protein 1 , a Wnt/ $\beta$-catenin pathway antagonist, was upregulated in knee SF and serum of OA patients and positively correlated with OA severity [43]. Analysis of microarray data from OA and normal 
cartilage also showed that the $\mathrm{Wnt} / \mathrm{Ca}^{+}$pathway is activated in OA [44]. The results of our in vivo experiments suggested that noncanonical Wnt ligands (Wnt5A, 5B, 7B) seemed to have an obvious promoting effect on OA progression, whereas the canonical Wnt ligand Wnt10A could improve the progress of OA (Fig. 5d). This phenomenon may indicate that our research on the role of the Wnt pathway in the pathogenesis of OA was insufficient, and more studies are needed to clarify this point.

Wnt signaling dysregulation was found to play a role in somatic stem cell dysfunction [14,15]. Noncanonical Wnt signaling appears to induce stem cell senescence, whereas canonical Wnt signaling has an antisenescence effect [7]. Florian et al. confirmed that the increased expression of Wnt5a in bone marrow caused an abnormal shift in Wnt signaling from the canonical to the noncanonical pathway, leading to stem cell senescence [14]. Weakening of canonical Wnt/ $\beta$-catenin signaling may result in the downregulated transcription of Trf2 and Tert, decreased telomerase activity, and shortened telomere repeat sequences [7]. Hoffmeyer et al. found that $\beta$-catenin directly bound the Tert promoter and upregulated telomerase activity to promote the proliferation of intestinal stem cells [45]. Based on our observations of enhanced noncanonical Wnt signaling and the large number of senescent SMSCs in the OA synovium, we aimed at exploring the possible causal association between Wnt signaling and senescence. The results of in vitro experiments showed that the noncanonical WNT ligands (WNT5A, 5B, and 7B) could, indeed, aggravate SMSC senescence, whereas the canonical WNT ligand WNT10A significantly decreased SMSC senescence (Fig. 5a). Recently, canonical Wnt signaling in the RA synovium was found to activate fibroblast-like synovial (FLS) cells, promote FLS cell proliferation, and simultaneously promote production of the inflammatory cytokines IL-6 and IL-8 [46]. We found that, in addition to the canonical WNT ligand WNT10A, the noncanonical WNT ligands WNT5A and $5 \mathrm{~B}$ could promote the degradation of cartilage matrix by SMSCs (Fig. 5a). In addition, WNT5B had a significant inhibitory effect on the chondrogenic differentiation of SMSCs and promoted a fibrotic phenotype in SMSCs (Fig. 5b and Supplementary Fig. S2). These results are consistent with the results of our histological analysis showing that WNT5A and 5B aggravated joint degeneration in an anterior cruciate ligament transection rat model and can partially explain the functional changes in SMSCs shown in Fig. 5. These results implicate WNT5A and 5B as potential targets of disease-modifying drugs [16-18]. Based on the observation of residual MSC failure in OA joints, Wnt inhibitors have recently completed phase I clinical trials as first-in-class drugs [17]. Although these results suggest the efficacy of Wnt inhibitors as DMOADs, WNT ligands and pathways with different effects have not been separately assessed. We found that some WNT ligands, such as WNT10A, are beneficial for the maintenance of partial SMSC function and cartilage integrity (Figs. 5b, c, 6 and Supplementary Fig. S2). Therefore, the development of more specific targeted Wnt inhibitors may be a future research direction.

There is a limitation of this study. Although we attempted to explore the relationship between WNT signaling dysregulation and stem cell dysfunction in OA synovium, only an extensive preliminary assessment has been provided thus far, and a definite causal relationship between the two was not confirmed in this study.

\section{Author Disclosure Statement}

No competing financial interests exist.

\section{Funding Information}

This work was supported by grants from the National Natural Science Foundation of China (81572150) and the Science and Technology Program of Hunan Province (2017SK2063).

\section{Supplementary Material}

Supplementary Figure S1

Supplementary Figure S2

Supplementary Table S1

Supplementary Table S2

\section{References}

1. Wiegant K, F Intema, PM van Roermund, RA Bartenvan, A Doornebal, HA Hazewinkel, FP Lafeber and SC Mastbergen. (2015). Evidence of cartilage repair by joint distraction in a canine model of osteoarthritis. Arthritis Rheumatol 67:465-474.

2. van der Woude J, K Wiegant, RJ van Heerwaarden, S Spruijt, PM van Roermund, R Custers, SC Mastbergen and F Lafeber. (2017). Knee joint distraction compared with high tibial osteotomy: a randomized controlled trial. Knee Surg Sports Traumatol Arthrosc 25:876-886.

3. McGonagle D, TG Baboolal and E Jones. (2017). Native joint-resident mesenchymal stem cells for cartilage repair in osteoarthritis. Nat Rev Rheumatol 13:719-730.

4. Jayasuriya CT, Y Chen, W Liu and Q Chen. (2016). The influence of tissue microenvironment on stem cell-based cartilage repair. Ann N Y Acad Sci 1383:21-33.

5. Cao X, P Luo, J Huang, C Liang, J He, Z Wang, D Shan, $\mathrm{C}$ Peng and S Wu. (2019). Intraarticular senescent chondrocytes impair the cartilage regeneration capacity of mesenchymal stem cells. Stem Cell Res Ther 10:86.

6. Fahy N, MM de Vries-van, J Lehmann, W Wei, N Grotenhuis, E Farrell, PM van der Kraan, JM Murphy, YM Bastiaansen-Jenniskens and GJ van Osch. (2014). Human osteoarthritic synovium impacts chondrogenic differentiation of mesenchymal stem cells via macrophage polarisation state. Osteoarthritis Cartilage 22:1167-1175.

7. Garcia-Velazquez L and C Arias. (2017). The emerging role of Wnt signaling dysregulation in the understanding and modification of age-associated diseases. Ageing Res Rev 37:135-145.

8. Lories RJ, M Corr and NE Lane. (2013). To Wnt or not to Wnt: the bone and joint health dilemma. Nat Rev Rheumatol 9:328-339.

9. Stampella A, S Monteagudo and R Lories. (2017). Wnt signaling as target for the treatment of osteoarthritis. Best Pract Res Clin Rheumatol 31:721-729.

10. van den Bosch MH, AB Blom, AW Sloetjes, MI Koenders, FA van de Loo, WB van den Berg, PL van Lent and PM van der Kraan. (2015). Induction of canonical Wnt signaling by synovial overexpression of selected Wnts leads to protease activity and early osteoarthritis-like cartilage damage. Am J Pathol 185:1970-1980. 
11. van den Bosch $\mathrm{MH}, \mathrm{AB}$ Blom, V Kram, A Maeda, S Sikka, Y Gabet, TM Kilts, WB van den Berg, PL van Lent, PM van der Kraan and MF Young. (2017). WISP1/CCN4 aggravates cartilage degeneration in experimental osteoarthritis. Osteoarthritis Cartilage 25:1900-1911.

12. Deshmukh V, H Hu, C Barroga, C Bossard, S Kc, L Dellamary, J Stewart, K Chiu, M Ibanez, et al. (2018). A small-molecule inhibitor of the Wnt pathway (SM04690) as a potential disease modifying agent for the treatment of osteoarthritis of the knee. Osteoarthritis Cartilage 26:18-27.

13. Van Camp JK, S Beckers, D Zegers and W Van Hul. (2014). Wnt signaling and the control of human stem cell fate. Stem Cell Rev Rep 10:207-229.

14. Florian MC, KJ Nattamai, K Dorr, G Marka, B Uberle, V Vas, C Eckl, I Andra, M Schiemann, et al. (2013). A canonical to non-canonical Wnt signalling switch in haematopoietic stem-cell ageing. Nature 503:392-396.

15. Woo DH, Q Chen, TL Yang, MR Glineburg, C Hoge, NA Leu, FB Johnson and CJ Lengner. (2016). Enhancing a Wnt-telomere feedback loop restores intestinal stem cell function in a human organotypic model of dyskeratosis congenita. Cell Stem Cell 19:397-405.

16. McAlindon TE and RR Bannuru. (2018). Osteoarthritis in 2017: latest advances in the management of knee OA. Nat Rev Rheumatol 14:73-74.

17. Yazici Y, TE McAlindon, R Fleischmann, A Gibofsky, NE Lane, AJ Kivitz, N Skrepnik, E Armas, CJ Swearingen, et al. (2017). A novel Wnt pathway inhibitor, SM04690, for the treatment of moderate to severe osteoarthritis of the knee: results of a 24-week, randomized, controlled, phase 1 study. Osteoarthritis Cartilage 25:1598-1606.

18. Chae WJ and A Bothwell. (2018). Canonical and noncanonical Wnt sdignaling in Immune Cells. Trends Immunol 39:830-847.

19. Liu S, E Zhang, M Yang and L Lu. (2014). Overexpression of Wnt11 promotes chondrogenic differentiation of bone marrow-derived mesenchymal stem cells in synergism with TGF-beta. Mol Cell Biochem 390:123-131.

20. Hulth A, L Lindberg and H Telhag. (1970). Experimental osteoarthritis in rabbits. Preliminary report. Acta Orthop Scand 41:522-530.

21. Xia J, EE Gill and RE Hancock. (2015). NetworkAnalyst for statistical, visual and network-based meta-analysis of gene expression data. Nat Protoc 10:823-844.

22. Gerwin N, AM Bendele, S Glasson and CS Carlson. (2010). The OARSI histopathology initiative - recommendations for histological assessments of osteoarthritis in the rat. Osteoarthritis Cartilage 18 Suppl 3:S24-S34.

23. Roelofs AJ, J Zupan, A Riemen, K Kania, S Ansboro, N White, SM Clark and C De Bari. (2017). Joint morphogenetic cells in the adult mammalian synovium. Nat Commun 8:15040.

24. Gullo F and C De Bari. (2013). Prospective purification of a subpopulation of human synovial mesenchymal stem cells with enhanced chondro-osteogenic potency. Rheumatology (Oxford) 52:1758-1768.

25. Hermida-Gomez T, I Fuentes-Boquete, MJ GimenoLongas, E Muinos-Lopez, S Diaz-Prado, FJ de Toro and FJ Blanco. (2011). Quantification of cells expressing mesenchymal stem cell markers in healthy and osteoarthritic synovial membranes. J Rheumatol 38:339-349.

26. Jones EA, A English, $K$ Henshaw, SE Kinsey, AF Markham, P Emery and D McGonagle. (2004). Enumeration and phenotypic characterization of synovial fluid multipotential mesenchymal progenitor cells in inflammatory and degenerative arthritis. Arthritis Rheum 50: $817-827$

27. Jones EA, A Crawford, A English, K Henshaw, J Mundy, D Corscadden, $\mathrm{T}$ Chapman, $\mathrm{P}$ Emery, $\mathrm{P}$ Hatton and D McGonagle. (2008). Synovial fluid mesenchymal stem cells in health and early osteoarthritis: detection and functional evaluation at the single-cell level. Arthritis Rheum 58:1731-1740.

28. Del Rey MJ, R Fare, A Usategui, JD Canete, B Bravo, M Galindo, G Criado and JL Pablos. (2016). CD271(+) stromal cells expand in arthritic synovium and exhibit a proinflammatory phenotype. Arthritis Res Ther 18:66.

29. Leijs MJ, GM van Buul, E Lubberts, PK Bos, JA Verhaar, MJ Hoogduijn and GJ van Osch. (2012). Effect of arthritic synovial fluids on the expression of immunomodulatory factors by mesenchymal stem cells: an explorative in vitro study. Front Immunol 3:231.

30. Vezina AR, A Lavoie-Lamoureux, JP Lavoie and S Laverty. (2013). Inflammatory stimuli differentially modulate the transcription of paracrine signaling molecules of equine bone marrow multipotent mesenchymal stromal cells. Osteoarthritis Cartilage 21:1116-1124.

31. Gomez-Aristizabal A, A Sharma, MA Bakooshli, M Kapoor, PM Gilbert, S Viswanathan and R Gandhi. (2017). Stage-specific differences in secretory profile of mesenchymal stromal cells (MSCs) subjected to early- vs late-stage OA synovial fluid. Osteoarthritis Cartilage 25:737-741.

32. Jeon OH, C Kim, RM Laberge, M Demaria, S Rathod, AP Vasserot, JW Chung, DH Kim, Y Poon, et al. (2017). Local clearance of senescent cells attenuates the development of post-traumatic osteoarthritis and creates a proregenerative environment. Nat Med 23:775-781.

33. Schultz MB and DA Sinclair. (2016). When stem cells grow old: phenotypes and mechanisms of stem cell aging. Development 143:3-14.

34. Cadigan KM and R Nusse. (1997). Wnt signaling: a common theme in animal development. Genes Dev 11: 3286-3305.

35. Dale TC. (1998). Signal transduction by the Wnt family of ligands. Biochem J 329 (Pt 2):209-223.

36. Ling L, V Nurcombe and SM Cool. (2009). Wnt signaling controls the fate of mesenchymal stem cells. Gene 433:1-7.

37. Zhou Y, T Wang, JL Hamilton and D Chen. (2017). Wnt/ beta-catenin signaling in osteoarthritis and in other forms of arthritis. Curr Rheumatol Rep 19:53.

38. MacDonald BT, K Tamai and X He. (2009). Wnt/betacatenin signaling: components, mechanisms, and diseases. Dev Cell 17:9-26.

39. Meo BP, L Xiao and MM Hurley. (2018). FGF23 regulates $\mathrm{Wnt} /$ beta-catenin signaling-mediated osteoarthritis in mice overexpressing high-molecular-weight FGF2. Endocrinology 159:2386-2396.

40. Tao SC, T Yuan, YL Zhang, WJ Yin, SC Guo and CQ Zhang. (2017). Exosomes derived from miR-140-5poverexpressing human synovial mesenchymal stem cells enhance cartilage tissue regeneration and prevent osteoarthritis of the knee in a rat model. Theranostics 7:180-195.

41. Lietman C, B Wu, S Lechner, A Shinar, M Sehgal, E Rossomacha, P Datta, A Sharma, R Gandhi, M Kapoor and PP Young. (2018). Inhibition of Wnt/beta-catenin signaling ameliorates osteoarthritis in a murine model of experimental osteoarthritis. JCI Insight 3: DOI: 10.1172/ jci.insight.96308. 
42. Hu S, G Mao, Z Zhang, $P$ Wu, $X$ Wen, W Liao and Z Zhang. (2019). MicroRNA-320c inhibits development of osteoarthritis through downregulation of canonical Wnt signaling pathway. Life Sci 228:242-250.

43. Theologis $\mathrm{T}, \mathrm{N}$ Efstathopoulos, V Nikolaou, I Charikopoulos, I Papapavlos, P Kokkoris, A Papatheodorou, N Nasiri-Ansari and E Kassi. (2017). Association between serum and synovial fluid Dickkopf-1 levels with radiographic severity in primary knee osteoarthritis patients. Clin Rheumatol 36:1865-1872.

44. Thorfve A, T Dehne, A Lindahl, M Brittberg, A Pruss, J Ringe, M Sittinger and C Karlsson. (2012). Characteristic markers of the WNT signaling pathways are differentially expressed in osteoarthritic cartilage. Cartilage 3: 43-57.

45. Hoffmeyer K, A Raggioli, S Rudloff, R Anton, A Hierholzer, I Del Valle, K Hein, R Vogt and R Kemler. (2012). Wnt/beta-catenin signaling regulates telomerase in stem cells and cancer cells. Science 336:1549-1554.

46. Miao C, J Chang, J Dou, Y Xiong and G Zhou. (2018). DNA hypermethylation of SFRP2 influences the pathology of rheumatoid arthritis through the canonical Wnt signaling in model rats. Autoimmunity:1-14.
Address correspondence to:

Song $W u, M D$

Department of Orthopaedics

The 3rd Xiangya Hospital

Central South University

Changsha 410013

China

E-mail: xy3ws1969@hotmail.com

Xи Cao, $M D$

Department of Orthopaedics

The 3rd Xiangya Hospital

Central South University

Changsha 410013

China

E-mail: hughcaoxu@hotmail.com

Received for publication November 2, 2019

Accepted after revision January 17, 2020

Prepublished on Liebert Instant Online January 22, 2020 